\title{
CARLO CATTANEO E LA «SCIENZA PENITENZIARIA»
}

\author{
Nota del m.e. MARIO PISANI (*)
}

(Adunanza del 9 marzo 2017)

SuNTO. - Il multiforme ingegno di Carlo Cattaneo ha avuto modo di applicarsi anche alla complessa ed antiquata disciplina della pena carceraria. In diverse occasioni, e muovendo da prospettive complementari, egli ha mirato a sottolineare la necessità di attuare un circolo virtuoso di intersezioni tra sperimentazione pratica ed elaborazione teorica, per dar vita, attraverso un approccio multidisciplinare, a concreti e positivi esiti di riforma.

ABSTRACT. - The manifold genius of Carlo Cattaneo had the chance also to put his mind to the complex and antiquated regulation of the punishment in prison. In various circumstances, and moving from complementary perspectives, he aimed to highlight the need to put in place a virtuous circle of intersections between practical experimentation and theoretical elaboration, in order to give birth, through a multidisciplinary approach, to concrete and positive reform outcomes.

\section{PREMESSA}

Nell'ultimo dei tre volumi che, nel 1846, aveva pubblicato sotto il generico titolo Alcuni scritti del dott. Carlo Cattaneo, il nostro autore aveva raccolto anche una serie di suoi contributi in materia carceraria che qualche anno prima aveva fatto stampare sul suo Politecnico. ${ }^{1}$

(*) Università degli Studi di Milano, Italy. E-mail: mariopisani.mp@libero.it

1 Alcuni scritti del dott. Carlo Cattaneo, vol. III, Milano, coi tipi Borroni e Scotti, 1846. (I vari contributi in materia carceraria sono ora reperibili anche nel vol. I, 
In una nota preliminare a tale raccolta, intitolata Della riforma penale, egli non mancava di indicare nominativamente gli autori, tutti stranieri meno uno, la "diligente lettura" dei quali aveva costituito la fase preparatoria del suo lavoro.

La breve nota è però importante per le sue caratterizzazioni d'ordine generale. "Chi sia persuaso che ogni scienza deve scaturire dai fatti - scriveva Cattaneo - non sarà tardo a credere che su l'ampia collezione che omai ne abbiamo, si potrebbe por mano a rifondere tutta l'oscura e malferma dottrina delle pene".

V'è da dire, a tale riguardo, che, invero, si trattava di un programma alquanto ambizioso, al quale, del resto, Cattaneo - ma non era mestier suo - non darà mai inizio. E piuttosto va dato risalto alle ulteriori e finali proposizioni nelle quali l'autore attingeva ad ancora più ampi ed alti orizzonti. "Questo studio dei fatti veri e positivi e inaspettati dell'umana natura nelle varie sue condizioni e vicissitudini, è appunto quello che il sapiente Romagnosi - il suo maestro -appellava filosofia civile; la quale deve preparare da lungi l'assiduo progresso e sviluppo delle sociali istituzioni". E risolutamente così aggiungeva: "E noi la vorremmo proposta nei tanti suoi argomenti a più fruttuosa occupazione delle scôle, le quali rimangono troppo vanamente a oziare intorno alli insolubili ritornelli d'una semibarbara ontologia".

Muovendo dunque da un settore assai circoscritto, ed ancora ben poco esplorato e soprattutto bonificato, delle esperienze collettive, quello delle carceri, in pochi tratti essenziali, distesi tra la teoria e la pratica, Cattaneo dava così espressione agli elementi di fondo delle sue ispirazioni ed aspirazioni ideali e della correlativa metodologia programmatica. $^{2}$

Firenze 1964, pp. 283-422, della raccolta degli Scritti politici di Carlo Cattaneo curata da Mario Boneschi). Nell'operare la raccolta, il nostro autore dichiarava di non aver "luogo a riprodurre" - in certo modo così accantonandoli - alcuni scritti precedenti, apparsi sui volumi V e VI del Politecnico: "una breve disputa intorno ai Pensieri di Valentino Pasini sul modo di proporre la questione penitenziaria; una notizia su li effetti del carcere segregante di Parigi; e un rapporto al Congresso di Lucca, redatto a nome d'una Commissione del Congresso di Padova".

2 Proprio evocando i concetti espressi in quelle pagine, Alessandro LEVI, Il positivismo di Carlo Cattaneo, Bari 1928, p. 16, scrive: "Non foss'altro per la sua gran fede nell'osservazione (...) C.C. merita d'esser detto un positivista". Il penalista Marcello FINZI parlava invece di "positivismo penale" di Cattaneo (in Riv. di diritto penale e sociologia criminale, 1900) con riferimento alle dottrine penalistiche della sopravveniente "Scuola positiva". 


\section{LA PROMISCUITÀ DEL CARCERE E L'ISTRUZIONE COME "SOMMO RIMEDIO" PREVENTIVO}

Se così scriveva nel 1846, nelle prime righe del suo contributo datato giugno 1840 , e posto sotto il titolo Delle carceri, ${ }^{3}$ non a caso l'autore già aveva avuto modo di richiamare il generale "proposito" che aveva animato tutta quanta l'iniziativa del Politecnico: quello - scriveva - "d'appianar ai nostri concittadini la cognizione di quella parte di vero, che dalle ardue ragioni della scienza può condursi a fecondare il campo della pratica”. E l'attacco dell'esordio si completava con l'evocazione della specifica circostanza ("della congiuntura") d'essersi egli trovato a "passare ad esame parecchie delle più recenti opere su la riforma carceraria", che gli aveva offerto l'occasione e lo stimolo "per tracciare in sommi capi lo stato d'una questione che tocca le più intime ragioni dell'arte sociale, e intorno a cui s'affaticano tanti illustri giureconsulti ed uomini di Stato in Europa e in America”.

La questione di cui parlava Cattaneo era poi quella del profilarsi di una "nuova scienza": “... fra le tenebre d'una dubiezza scrutatrice e feconda si svolgono - scriveva - le forme d'una nuova scienza, che, trasandando le odiose idealità dell'espiazione, e mirando solo a estinguere il fenomeno criminoso, assoggetta a vasto processo esperimentale tutti i fatti dell'immoralità”. E il discepolo di Romagnosi aggiungeva: “ ... Lo studio del diritto penale non può fare un passo, se non muovendo dalle nuove generalità nelle quali la scienza carceraria va ordinando la moltitudine delle osservazioni".

Lo studio e l'osservazione spaziano dunque entro una geografia di continenti: perché, infatti - notava Cattaneo - si tratta di "ragionare con impassibilità scientifica sul destino d'un millione e più di sciagurati, ch'entrano ogni anno nelle carceri d'Europa e d'America; molte milliaia dei quali vi scendono come in un sepolcro di viventi, o n'escono solo per salire al patibolo".

La grande ampiezza e l'indeterminatezza analitica degli orizzonti di ricerca propizia, ed in qualche modo impone all'autore, un affresco piuttosto movimentato, di dati e di riflessioni, tra i quali non è sempre agevole discernere le messe a punto dei materiali oggetto di studio dalle personali valutazioni dello scrivente che li mette a profitto.

3 Alcuni scritti, cit., pp. 80-116. 
Alcuni elementi di giudizio emergono tuttavia chiaramente dallo specifico contributo qui in discorso. Muovendo, peraltro con eccesso di semplificazione, dall'ipotesi radicale di un dualismo senza varianti, Cattaneo, che anni prima aveva enfaticamente sottolineato 1' "immenso potere penale della solitudine", esprimeva la sua netta preferenza a favore del regime della "rigida segregazione" della "cella solitaria" rispetto all'alternativa della "promiscuità fra i detenuti":4 una segregazione, ad ogni modo, la quale, "più ancora che ai condannati - così, ad ogni modo, egli specificava con grande avvedutezza - è giusta e utile e provida ai giudicandi, che la legge o deve assegnare alla pena, o rendere all'onore o alla libertà puri come li trovò, non allacciati da turpi conoscenze, non avviliti e irritati da illegittimi e brutali castighi".

Di non minore avvedutezza e lungimiranza il nostro autore dava prova quando, echeggiando il Beccaria, pur non nominandolo, ${ }^{6}$ indicava l'istruzione - non esclusa quella "compartita, se non altro, nel carcere" - come il "sommo rimedio al male". Ma "l'istruzione data nel carcere giunge troppo fuor di tempo. Bisogna invece - opportunamente veniva a chiarire - che la società provveda prima, e supplisca nei fanciulli miserabili all'impotenza o incuria o assenza dei genitori; e con li asili delli infanti, e i ricoveri delli orfani e abbandonati, e le scôle delli artigiani, tolga l'età innocente alla vorace depravazione".

E poco oltre Cattaneo veniva a concludere sul punto: "La maggior parte delle prigioni d'Europa e d'America attende ancora la gran riforma, a cui si richiede tempo, pensieri e tesori".

4 Non mancherà poi chi, alla distanza, muoverà aspra critica a Cattaneo, con opposta accentuazione di tono estremistico: v. BELLONI, Cattaneo criminalista, Milano 1943, p. 42, a giudizio del quale la "carcerazione segregante" altera talmente la persona "da renderla disadatta più che mai alla vita sociale", cosicchè "la solitudine allora non val meglio della convivenza depravatrice". La pratica carceraria ha conosciuto peraltro una pluralità di "sistemi": v., ad esempio, BELTRANI SCALIA, La riforma penitenziaria in Italia - Studi e proposte, Roma 1879, p. 127, che ne enunciava tre: quello "di segregazione continua o cellulare"; quello di Auburn (cioè "separazione dé detenuti durante la notte ma lavoro in comune durante il giorno con l'obbligo del silenzio"); quello "progressivo e graduale".

5 Alcuni scritti, cit., pp. 109.

6 BECCARIA, Dei delitti e delle pene, $\mathbb{X}$ XLV: "Finalmente il più sicuro ma più difficil mezzo di prevenire i delitti si è di perfezionare l'educazione". 


\section{LA "STRETTA CONNESSIONE" TRA LA "SCIENZA PENALE" E LA “MEDICINA”.}

Tempo e pensieri per la riforma auspicata il nostro autore avrà modo di dedicare qualche anno dopo, per dar corso alla pubblicazione sul Politecnico, nel 1842, di un contributo sul vetusto tema Della deportazione - che, dichiarerà più tardi Cattaneo, "ebbe occasione da un discorso di Sir William Molesworth" - e l'anno dopo, e sempre sul Politecnico, di un saggio dal titolo Delle galere, "ad esame dell'opera intitolata" Les forçats considerés sous le rapport physiologique, moral et intellectuel, observés au bagne de Toulon, di certo dottor Lauvergne.

Questo secondo lavoro si segnala, in particolare, per una nota pionieristica di originalità, per il fatto che Cattaneo, sempre ispirandosi alla metodologia induttiva dell' "osservazione", dava grande risalto alla "stretta connessione della scienza penale e della medicina" che col tempo diceva - è divenuta "sempre più manifesta". Dall' "osservazione medica", intesa nel senso amplissimo che l'autore verrà poi specificando, è dato sperare - egli scriveva - piuttosto che non "dalle deduzioni dei giureconsulti", un certo qual "grande e sùbito incremento della dottrina criminale". E ciò nel senso che "i fatti che il medico" - si vorrebbe dire il plurisapiente e saggio esploratore dei corpi e delle menti - è in grado di raccogliere non si restringono ( $\mathrm{ma}$ vi è anche un'implicita esortazione) "solo al governo del carcere e alla vita dei miseri reclusi”.

Piuttosto e invece essi "si riferiscono alla più intima parte della ragion penale", ossia - e ancora una volta Cattaneo si professava fedele allievo di Romagnosi, e seguace della teorica da questi espressa nella Genesi del diritto penale ${ }^{8}$ " all'esplorazione di quella spinta criminosa, da cui dipende la scelta e misura delle pene, come nell'arte militare dalla natura e potenza delle armi offensive dipendono le condizioni della difesa".

A questo quadro programmatico sottendeva pur sempre un'impostazione di carattere filosofico. Vi sono senz'altro - rilevava infatti il nostro autore - uomini "per natura feroci, perversi, proclivi al furor delle passioni, come altri al contrario fin dal seno materno sembrino

7 Per il testo dei due saggi v. Alcuni scritti, cit., pp. 117-139; pp. 140-157.

8 Sul tema v. NUVOLONE, Delitto e pena nel pensiero di G.D. Romagnosi (1961), ora in Trent'anni di diritto e procedura penale, vol. I, Padova 1969, p. 350. 
temperati a mansuetudine e benevolenza, sicchè a tenerli sulla diritta via non è certamente mestieri atterrirli col volto del carnefice e col suono delle catene. Codeste varietà delli umani individui - proseguiva Cattaneo - dal medico possono essere osservate: ma l'uomo dei metafisici, i quali sogliono muovere appunto dalle osservazioni generali della metafisica, si riduce a cifra unica e costante, a invariabile astrazione".

E poche pagine dopo troviamo scritto, con riferimento all'autore commentato: "Diremo dunque che utile ne pare il pensamento di notare con esattezza medica tutti i fatti morali e corporei dell'individuo malfattore; e siamo persuasi che da queste particolari istorie, raccolte in più luoghi e presso diverse nazioni con fedeltà, debba scaturire qualche induzione su la spinta criminosa in certe nature eccezionalmente infelici”. Ne discende la conseguenza - di portata alternativa, e innovativa, rispetto all'antica logica del carnefice e delle catene - che, si noti, "una parte della controspinta verrà pietosamente rassegnata alla cura del medico; e forse - aggiungeva Cattaneo - una reclusione preventiva e scevra d'ogni penalità verrassi palesando come unica via di premunire la società da certi delitti, che possono piuttosto tenersi eruzioni d'insania, che atti di pensata malvagità".

E' così aperta, per il futuro, la strada verso il diritto penale della prevenzione.

\section{LA MATERIA CARCERARIA E LA RIFORMA DELL'INSEGNAMENTO PUBBLICO}

In altra occasione s'era già avuto modo di riferire intorno all'invito che, nel gennaio 1848, il vice-governatore O'Donnel aveva rivolto al nostro Istituto Lombardo, a quei tempi organo consultivo dell'attività di governo, a voler predisporre un progetto di riforma dell'ordinamento scolastico relativo alle scuole elementari e ginnasiali, oltre che alle "case di educazione".

Non foss'altro la nota di brevità, allora adottata, ci consente più facilmente di riprendere l'argomento, allo scopo di meglio illustrare

9 PISANI, Carlo Cattaneo, dottore «in utroque jure», in Dall'Università di Pavia - Figure emergenti tra' 800 e '900, Pavia 2016, p. 13. 
qualche dettaglio concernente la particolare tematica che si viene ora esaminando.

Va allora ricordato che al Lombardo veniva sùbito costituita una commissione, della quale erano chiamati a far parte Gabrio Piola, Pompeo Litta, Francesco Rossi, Francesco Restelli, mentre a Carlo Cattaneo, in quei giorni promosso da membro "non pensionato" a membro "pensionato" dell'Istituto, veniva affidato il compito di relatore: un compito in certo modo poi facilitatogli dalla presentazione di ben quaranta rapporti settoriali inoltrati dai colleghi.

Alla fine fu approvata e stampata una relazione di trenta pagine, intestata: "Sull'ulteriore sviluppo del pubblico insegnamento in Lombardia", ${ }^{10}$ che fu oggetto di discussione per alcune sedute. ${ }^{11} \mathrm{E}$ ciò fino a che, ad interromperla, fu addirittura l'insurrezione lombarda delle cinque giornate entro la quale la sorte, com'è ben noto, affiderà a Cattaneo ben altre e più celebri incombenze.

Quel che qui preme sottolineare, ai fini del nostro discorso in tema di riforma carceraria, è che questo tema si fa spazio, in modo anche imprevedibile, in molti e disparati capitoli della relazione approntata secondo intendimenti che senz'altro erano da riportarsi, in correlazione con i suoi qui richiamati studi pregressi, all'insigne relatore.

A proposito delle scuole elementari, nel pur breve paragrafo (n. 6) dedicato alle "Scuole dei carcerati", il relatore-riformista trovava modo, alla fine, di così esprimersi: "Noi vorremmo dalla misericordia e religione del Monarca invocare eziandio che venisse insinuato ai Magistrati qualche riguardo al pudore degli adolescenti, sul punto almeno di non tradurli a piedi ogni giorno nelle ore meridiane da uno ad altro carcere". Ed ancora, echeggiando qualche già richiamata messa a punto del Cattaneo-scrittore, ma qui anche più opportunamente posta in rapporto con lo specifico comparto minorile: "Noi vorremmo dalla commiserazione del Regnante invocare finalmente qualche provvedimento alle

10 Il sottotitolo della relazione era così formulato: "Riassunto di varie Memorie presentate all'I.R. Istituto di parecchi Membri onorari ed effettivi e Soci corrispondenti, in seguito a deliberazione 13 gennaio 1848, e per cura di speciale Commissione a ciò deputata, onde poi farne oggetto d'ulteriori deliberazioni dell'Istituto": v. in Tutte le opere di Carlo Cattaneo, a cura di L. Ambrosoli, vol. IV, Milano 1967, p. 3 ss., e poi le note illustrative, nelle pp. 928 ss.

11 L. AMBROSOLI, La discussione dell'Istituto Lombardo sul progetto per lo sviluppo dell'insegnamento in Lombardia, in Critica storica, 1965, p. 781. 
sozze tenebre del carcere promiscuo, ove si confondono in una voragine gli estremi dell'innocenza e della depravazione. Il carcere da scuola d'ogni abominio può divenire scuola d'onesta operosità".

Va poi aggiunto che, andando oltre il perimetro dell'invito rivolto all'Istituto, riguardante le varie scuole d'ordine inferiore, la Commissione si trovò ad occuparsi, e non poco, anche delle scuole superiori, e, per l'appunto delle "Scuole Universitarie".

Occupandosi con particolare attenzione della Facoltà legale, il relatore iniziava con il sottolineare il "manifesto oscuramento" nel quale versava lo studio dell'alta giurisprudenza, e passava poi a delineare, disciplina per disciplina, i contenuti migliorativi delle varie materie di insegnamento, a muovere $(\$ 38)$ dal Diritto romano.

Alcuni paragrafi dopo la relazione brevemente si intratteneva sulla materia, pur senza evocare Romagnosi, designata come Filosofia civile, per dire della ancora carente preparazione filosofica, "nobil parte della qual sarebbe la logica giuridica". E così, con singolare chiaroveggenza, si spiegava: “... ossia la dottrina delle prove, necessario fondamento della razionale procedura civile e criminale", mentre invece nelle sue "particolarità materiali", la Procedura - concludeva pur senza rassegnazione - "non s'impara mai in effetto nelle Università, ma nella esperienza degli affari”.

Passando, poi, in poche righe - ma è ciò che più conta - a trattare della materia del Diritto penale $(\$ 42)$, sempre il Cattaneo - possiamo pure dire così, e non solo per semplicità di discorso - veniva a scrivere che esso "non dovrebbe studiarsi solo a illustrazione delle leggi sin qui sancite", e dunque, diremmo noi oggi, in termini di diritto positivo vigente, bensì "in vista alle nuove dottrine penitenziarie, le quali - si spingeva a scrivere il nostro autore - tendono a immutar fra poco tutto il calcolo delle pene".

$\mathrm{Al}$ di là di tutto questo, che di per sé non era poi programma di poco conto, ancora una volta Cattaneo non perdeva l'occasione per mettere in campo la sua generalissima ispirazione di progresso civile. "La gioventù - concludeva sùbito dopo - deve prepararsi a quei pensieri che l'attendono in seno alla società".

E ciò anche e perfino parlando $(\$ 44)$ della Eloquenza in quanto disciplina specifica della Facoltà legale. "La gioventù - scriveva il relatore - dovrebbe poi esercitarsi nell'arte della parola, e perché nel paese nostro è gravemente decaduta, e perché giova prevedere il caso nel quale al Sovrano piacesse di assentire al voto che invoca, nell'ammini- 
strazione della giustizia, delle forme pubbliche, come nella rimanente Italia e nei più colti regni d'Europa”.

\section{IL SISTEMA PENALE E LA SACERTÀ DEL "PRINCIPIO PENITENZIARIO"}

Nella prefazione pubblicata sul Politecnico del 1860 - si trattava del volume IX - Cattaneo "metteva a fuoco i principali temi che sarebbero stati trattati dalla seconda serie della rivista". ${ }^{12}$

Ma in quella sede, a proposito del "sistema penale" il nostro autore presentava ben più di una sintetica anticipazione. ${ }^{13}$ Di esso diceva trattarsi di "un'appendice e un supplemento alla morale", e come tali soggetti alle diversità dell'atteggiarsi di quest'ultima nei diversi contesti sociali. Passava poi subito ad evocare, insieme a Beccaria, "il tetro argomento della pena di morte" intesa come "punto capitale di tutto l'ordine delle pene".

A tale proposito contestava vigorosamente sia gli ostinati difensori della medesima, che osavano parlare della posizione degli abolizionisti addirittura come "umiliante per la coscienza umana", sia i "casisti del patibolo" (abolire la pena di morte - dicevano - ma "caso per caso"), sia, ancora, gli attendisti, i quali invece si limitavano ad auspicare la totale abolizione del patibolo, "in un'epoca che si può sperare non lontana”. Ma quando, opponeva Cattaneo, "verrà quest'epoca? E a quali segni potranno riconoscerla i popoli?".

Muovendo da questi rilievi radicali, con fervore polemico il nostro autore trascorreva sùbito, spostando con impeto il quadro di riferimento, ad evocare i "cadaveri viventi, dissepeliti dagli aridi sotterranei di Palermo e di Napoli", e di contro a rendere elogio a quei "filantropi" impegnati "a trasformare l'inferno del carcere e della galera, risonante di catene e di flagelli e di bestemmie, e divorato dagli animali immondi, dal lezzo, dal tifo, dalle lascivie, in case di lavoro e d'insegnamento e di lettura, con tutte le condizioni umanamente possibili di salubrità, di quiete, e diremo anche, d'individuale dignità".

Ai difensori delle pratiche vigenti Cattaneo rigorosamente oppo-

12 LACAITA, in CARLO CATTANEO, I problemi dello Stato italiano, Milano 1966, p. 53. (Nel frattempo il Consiglio di Stato elvetico il 10 ottobre 1852 aveva nominato Cattaneo professore di filosofia nel liceo cantonale di Lugano).

13 V. ibid., pp. 67-69. 
neva che essi mettevano "in sospetto e in odio ciò che vi è di più sacro e santo nel mondo". E ciò in quanto - proseguiva l'autore con un'enunciazione memorabile - "il principio penitenziario mira a trasformare, per quanto può sperarsi, l'antica e perpetua scôla del delitto e dell'infamia in una scôla d'industria e d'ordine e in un ravvivamento della ragione e della coscienza". Ed aggiungeva, in polemica con "La Perseveranza", un giornale fondato quell'anno: "E' un dolore per noi, che abbiamo sempre dato larga parte dei nostri studii a sì grave argomento, vedere come si possa prodigare il denaro e l'ingegno in questa triste impresa di suscitare ostacoli alle più solenni opere di ragione e d'incivilimento, e avvelenare e imbarbarire l'opinione".

\section{LA "SCIENZA PENITENZIARIA" E IL RIORDINAMENTO DEGLI STUDI SCIENTIFICI}

Un altro ed ultimo contributo di Cattaneo alla materia (al "sì grave argomento") è rappresentato dalla lettera al senatore Matteucci, ministro della Pubblica Istruzione, pubblicata nel 1862 sul volume XII del Politecnico, sotto il titolo "Sul riordinamento degli studi scientifici" ${ }^{14}$ A distanza di pochi giorni dalla presentazione, avvenuta il 15 giugno 1861, di un disegno di legge in materia, il ministro aveva chiesto a Cattaneo che gli volesse comunicare "liberamente" le sue "objezioni, modificazioni e aggiunte".

All'intendimento del ministro di delineare "un insegnamento uniforme in tutte le università e facultà d'Italia", il Cattaneo opponeva che, invece, il "principio che abbisogna alle facultà italiane, è adunque ciò che in economia si chiama divisione del lavoro (...). La sintesi sarà l'Italia. - aggiungeva - La sintesi non è ripetizione, non è l'uniformità; ma è la più semplice espressione della massima varietà".

Affrontando poi - ma è quanto più interessa alla nostra ricognizione in materia carceraria - il tema dell'insegnamento universitario delle scienze mediche, Cattaneo auspicava che venissero approntati "nuovi lumi alle vetuste università", anche attraverso la predisposizione di "brevi corsi speciali". ${ }^{15}$

$\begin{array}{ll}14 & \text { V. ibid., pp. } 123-146 . \\ 15 & \text { V. ibid., pp. } 137 .\end{array}$ 
Faceva il caso di una "grande scôla d'oculistica", che "viene assegnata dalla natura a qualche gran città nei climi infestati dalle oftalmie". Faceva poi il caso dell'"asilo d'invalidi", che "sarà sempre un museo vivente per la chirurgia operativa". E sùbito dopo, in parallelo a tale casistica - fedele com' era ai criteri della metodologia induttiva che deve ispirare le correlazioni, per una sorta di circolo virtuoso, tra la sperimentazione pratica e l'elaborazione teorica, e tra quest'ultima e la fecondità della sua traduzione nell'insegnamento - il nostro autore aveva modo di aggiungere: "Un popoloso carcere è la naturale sede di quella scienza penitenziaria che ondeggia tra il diritto, la medicina, e la psicologia (...). Anche di quest' altissimo ramo si potrebbe dare, a intervallo d'anni, un corso speciale". Ed utile, anzi essenziale, risultava poi l'ulteriore specificazione: " $\mathrm{E}$ fra i giovani che lo seguissero, si dovrebbe a preferenza scegliere i medici carcerarj e i direttori delle prigioni”.

\section{SPUNTI CONCLUSIVI}

Nel corso dei decenni sopravvenuti, la cosiddetta (e impropriamente detta) scienza penitenziaria ${ }^{16}$ ha finito di ondeggiare - come allora diceva Cattaneo - tra il diritto, la medicina e la psicologia.

Essa è venuta progressivamente affinandosi, e in qualche modo assestandosi su basi di più razionale complessità, concretatasi, per un verso, nell'articolazione positiva e composita, ex novo, in Italia ma non solo, di un sistema di Diritto penitenziario,${ }^{17}$ che da pochi anni costituisce anche materia su larga scala insegnata nelle nostre Università; per altro verso mediante la creazione di diverse figure professionali e istituzionali,

16 L'improprietà, ricorrente nel vario modularsi che di quella aggettivazione continua a venirci proposto non solo in Italia, consiste nell'implicita e simmetrica correlazione, per così dire vetero-testamentaria, della penitenza come risposta al peccato. $\mathrm{E}$ ciò quando invece il superamento della nozione del delitto come peccato aveva rappresentato uno dei più ragguardevoli contributi che Beccaria aveva apportato alla storia della civiltà.

17 Sul diritto penitenziario come "realizzazione positiva della scienza penitenziaria", in quanto costituente "il sistema giuridico dell'esecuzione [penale] accolto dalla legislazione di un determinato paese", v. NOVELLI, L'autonomia del diritto penitenziario, in Riv. di dir. penit., 1933, p. 49; SIRACUSA, Istituzioni di diritto penitenziario, Milano 1935, p. 8 (quest'A. annovera Cattaneo - p. 3 - tra i non pochi cultori di una "scienza delle prigioni", la quale, a suo giudizio, "più che scienza fu arte"). 
operanti quali coadiutrici sia a livello giurisdizionale che a livello della pratica esecutiva. E' venuta così proliferando quella poliedrica ed unitaria figura del "medico", il cui apporto alla legislazione e alla giurisprudenza Cattaneo aveva presagito come necessario e imprescindibile: apporto oggi mediato soprattutto dagli studi e dalle risorse, oltre che della psicologia e della psichiatria, della criminologia e della sociologia.

Ma il contributo di Cattaneo, antesignano e propugnatore, come s'è visto, dell'integrazione di scienze ed esperienze, anche ai nostri occhi disincantati appare come storicamente significativo e meritorio.

Ed ancora una volta ci fa pensare alla celebre frase di Bacone che Beccaria aveva collocato all'ingresso di "Dei delitti e delle pene": nelle questioni di qualche particolare difficoltà non c'è da attendersi che vi sia qualcuno che, insieme, semini e raccolga, sed preparatione opus est, ut per gradus maturescant. 